UK. ${ }^{4}$ Dept of Medicine, The Ottawa Hospital Research Institute, University of Ottawa, Ottawa, Canada. ${ }^{5}$ Institute for Lung Health, NIHR Respiratory Biomedical Research Unit, University of Leicester, Leicester, UK.

Correspondence: Mona Bafadhel, Dept of Respiratory Medicine, Nuffield Dept of Medicine, NDM Research Building, University of Oxford, Old Road Campus, Oxford, OX3 7FZ, UK. E-mail: mona.bafadhel@ndm.ox.ac.uk

Received: April 022014 | Accepted: April 212014 | First published online: June 122014

Support statement: This report is independent research arising from a Post-Doctoral Fellowship held by M. Bafadhel supported by the National Institute for Health Research (grant number NIHR-PDF-2013-06-052). The views expressed in this publication are those of the authors and not necessarily those of the NHS, the National Institute for Health Research or the Department of Health.

Conflict of interest: Disclosures can be found alongside the online version of this article at erj.ersjournals.com

\title{
References
}

1 Vestbo J, Hurd SS, Agustí AG, et al. Global strategy for the diagnosis, management, and prevention of chronic obstructive pulmonary disease: GOLD executive summary. Am J Respir Crit Care Med 2013; 187: 347-365.

2 Walters JA, Gibson PG, Wood-Baker R, et al. Systemic corticosteroids for acute exacerbations of chronic obstructive pulmonary disease. Cochrane Database Syst Rev 2009; 1: CD001288.

3 National Clinical Guideline Centre. Chronic obstructive pulmonary disease: management of chronic obstructive pulmonary disease in adults in primary and secondary care. London, National Clinical Guideline Centre, 2010. http:/guidance.nice.org.uk/CG101/Guidance/pdf/English

4 Leuppi JD, Schuetz P, Bingisser R, et al. Short-term vs conventional glucocorticoid therapy in acute exacerbations of chronic obstructive pulmonary disease: the REDUCE randomized clinical trial. JAMA 2013; 309: 2223-2231.

5 Bafadhel M, McKenna S, Terry S, et al. Blood eosinophils to direct corticosteroid treatment of exacerbations of chronic obstructive pulmonary disease: a randomized placebo-controlled trial. Am J Respir Crit Care Med 2012; 186: $48-55$.

6 Davies L, Angus RM, Calverley PM. Oral corticosteroids in patients admitted to hospital with exacerbations of chronic obstructive pulmonary disease: a prospective randomised controlled trial. Lancet 1999; 354: 456-460.

7 Aaron SD, Vandemheen KL, Maltais F, et al. TNF $\alpha$ antagonists for acute exacerbations of COPD: a randomised double-blind controlled trial. Thorax 2013; 68: 142-148.

8 Anthonisen NR, Manfreda J, Warren CP, et al. Antibiotic therapy in exacerbations of chronic obstructive pulmonary disease. Ann Intern Med 1987; 106: 196-204.

9 Bafadhel M, McKenna S, Terry S, et al. Acute exacerbations of chronic obstructive pulmonary disease: identification of biologic clusters and their biomarkers. Am J Respir Crit Care Med 2011; 184: 662-671.

\section{Polypharmacy in patients hospitalised for acute exacerbation of COPD}

\section{To the Editor:}

Chronic obstructive pulmonary disease (COPD) imposes a great burden on health systems, and the expense will increase in the years to come [1]. Medication represents a large proportion of COPD-related costs [2], and has increased by $170 \%$ over the past 20 years [3]. Inpatients with an acute exacerbation of COPD and more than four diagnoses after admission are prescribed significantly more drugs and present with polypharmacy more frequently [4]. It is well known that polypharmacy is associated with greater occurrence of adverse effects [5] and worse treatment adherence [6]. It also contributes to greater healthcare expense [7].

This study seeks to determine the prevalence of polypharmacy in patients hospitalised with an acute exacerbation of COPD, as well as the factors associated with its occurrence.

The ECCO study is an observational, cross-sectional and multicentre study, with participation from 26 Internal Medicine Departments throughout Spain. Detailed features of the study have been reported elsewhere [8]. Each researcher included, consecutively, every patient admitted for an acute exacerbation of COPD they attended between January 1, 2007 and December 31, 2008. COPD was diagnosed using spirometry [9] in a stable condition prior to admission. Information gathered included age, sex, smoking history, previous admissions for COPD and number of acute exacerbations of COPD over the previous 12 months. The Charlson index was used to assess associated comorbidities, but other conditions prevalent in COPD not included in that index were also noted. Basal dyspnoea prior to admission was assessed using the modified (five point) Medical Research Council (mMRC) scale. Polypharmacy was defined as the chronic concurrent use, more than 3 months prior admission, of five or more drugs [10], and excessive 
polypharmacy as the use of 10 or more medications. The study was approved by the Clinical Research Ethics Committee of the coordinating centre (Hospital de la Vega Baja, Orihuela, Alicante, Spain) and all patients signed an informed written consent form. Quantitative data are presented as mean \pm SD. Comparisons of continuous variables between the two groups were performed using the t-test or the Mann-Whitney U-test whenever variables did not show a normal distribution. Categorical variables are presented as absolute frequencies and percentages. Comparisons between both groups were made using Chi-squared and Fisher's exact tests. To compare proportions between drugs prescribed before the admission and at the time of discharge, a Z-proportion test was carried out. A multivariate logistic regression model was constructed using the variables associated with polypharmacy at discharge in the univariate analysis with a p-value $<0.10$. In all analyses $p$-values $<0.05$ were considered statistically significant.

We included 398 patients, 353 males and 45 females, with a mean \pm SD age of $73.7 \pm 8.9$ years. At the time of admission, the mean \pm SD number of chronic drugs per patient was $5.0 \pm 2.6$. A total of 224 (56.3\%) patients presented polypharmacy and $22(5.5 \%)$ excessive polypharmacy. Compared with patients without polypharmacy, those with polypharmacy were more frequently ex-smokers $(75.4 \%$ versus $68.4 \%, \mathrm{p}=0.0005)$ and used long-term oxygen therapy $(41.5 \%$ versus $30.5 \%, \mathrm{p}=0.02)$. They scored higher in the Charlson index (2.8 versus $2.3, \mathrm{p}=0.004)$, had more comorbidities, and more severe dyspnoea measured with the mMRC scale (3.7 versus $3.5, \mathrm{p}=0.02$ ), although they showed no differences regarding their COPD Global Initiative for Chronic Obstructive Lung Disease stage.

21 patients died during hospitalisation, six (1.5\%) of them without and $15(3.8 \%)$ with polypharmacy $(\mathrm{p}=0.15)$. At discharge, the number of drugs for chronic conditions, excluding systemic corticosteroids or antibiotics administered for the current COPD exacerbation, was $6.6 \pm 2.4$ and had increased in 272 (72.1\%) patients. $296(78.5 \%)$ patients presented polypharmacy and $44(11.7 \%)$ excessive polypharmacy. Factors independently associated with polypharmacy at discharge were polypharmacy at admission, a lower post-bronchodilator forced expiratory volume in $1 \mathrm{~s}$ (FEV1) \% predicited and presence of heart failure (table 1).

There were changes in respiratory drug use before admission and at discharge. The use of combinations of long-acting $\beta_{2}$-agonists with inhaled corticosteroids $(50.7 \%$ versus $69.0 \%, p=0.0003)$, inhaled anticholinergics $(71.9 \%$ versus $87.5 \%, \mathrm{p}=0.0001)$, diuretics $(42.4 \%$ versus $51.7 \%, \mathrm{p}=0.01)$, proton pump inhibitors $(48.0 \%$ versus $68.7 \%, \mathrm{p}=0.0008)$ and beta-blockers $(3.9 \%$ versus $8.0 \%, \mathrm{p}=0.02)$ had increased.

Our study shows that patients hospitalised with an acute exacerbation of COPD have been prescribed a mean of five drugs before their admission, and that the number increases after their discharge. Comorbidities, especially heart failure, and impaired lung function are the factors associated with a greater use of medication.

Polypharmacy is a frequent problem in elderly patients. A study associated having more than four diagnoses with using seven or more drugs [4]. The same association was observed with certain diagnoses, such as COPD, heart failure, diabetes mellitus, hypertension, ischaemic heart disease and renal failure. Our study has shown similar associations. Old age and comorbidities are frequent among patients of internal medicine departments, and usually these patients are more drug-dependent. In the Registro Politerapi SIMI, carried out in Italian internal medicine departments, COPD was independently associated with polypharmacy [11].

TABLE 1 Factors associated with polypharmacy at discharge

Univariate analysis

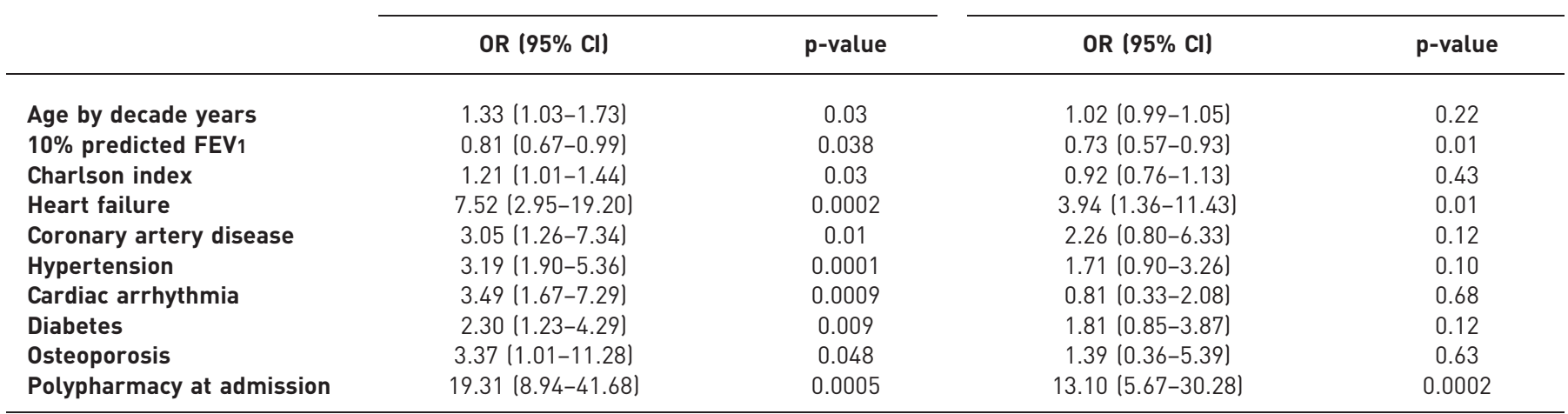

FEV1: forced expiratory volume in $1 \mathrm{~s}$. 
COPD-heart failure and COPD-coronary artery disease clusters were also strongly associated with polypharmacy [11]. In our study, these two comorbidities, heart failure and coronary artery disease, were present in $27 \%$ and $17 \%$ of COPD patients. A lower FEV1 \% predicted has also been associated with polypharmacy. These results were to be expected, since international clinical practice guidelines recommend using a variety of drugs for the treatment of both COPD and cardiovascular diseases. However, there are some difficulties in adapting and following the guidelines for patients suffering from multiple diseases [12]. Recommendations in the guidelines are based on clinical trials, and it is well known that trials usually exclude elderly patients with multiple diseases [13]. Furthermore, the use of more medication does not necessarily result in better health outcomes. In the case of COPD patients with a reduced life expectancy, rational prescription is paramount [14], assessing interactions, risks and benefits, and prioritising comfort, symptom control and quality of life.

Clinical inertia is a big challenge in chronic diseases including COPD, both from the diagnostic and the therapeutic angle [15]. Up to one third of all patients diagnosed with COPD are not treated. Our study shows an increase in the number of respiratory drugs prescribed at discharge, especially inhaled anticholinergics and combinations of long-acting $\beta_{2}$-agonists with inhaled corticosteroids. However, we cannot rule out that a negative clinical inertia possibly led to maintenance at discharge of drugs unnecessarily prescribed before admission. The mean number of drugs increased from 5 before admission to 6.6 at discharge, and polypharmacy before admission was the major determining factor of polypharmacy at discharge.

Our study has some limitations. First, patients were recruited in internal medicine departments. In these departments COPD patients are older and suffer from more comorbidities than those attended in respiratory departments. This might limit the widespread generalisation of our conclusions outside this setting. Secondly, female patients were scarcely present in the study. This is common in other studies carried out in Spain, where the prevalence of smoking in females only started increasing after 1960. Thirdly, patients were recruited during hospitalisation for an acute exacerbation of COPD. The frequency of polypharmacy and the profile of drug prescription probably differ between COPD patients with frequent acute exacerbations of COPD and patients without any acute exacerbation of COPD. In the days or weeks prior to their admission, patients with an acute exacerbation of COPD may have experienced increasing symptoms and perhaps been prescribed new drugs. It is also possible that at discharge they were prescribed antibiotics, corticosteroids or other drugs just for a few days, only to have them removed later in a control visit. However, to limit this factor, only drugs prescribed for chronic conditions were considered and the use of antibiotics, systemic steroids or mucolytics prescribed for the current exacerbation were excluded.

In summary, our study concludes that polypharmacy is frequent among patients hospitalised for an acute exacerbation of COPD and that it is associated with polypharmacy prior to admission, greater spirometric severity and a larger number of comorbidities, particularly heart failure. We propose that the presence of polypharmacy should be assessed in all COPD patients, and that whenever it is present, pharmaceutical care measures should be included in relation to these patients, in order to maximise adherence and reduce the possibility of adverse effects and drug interactions.

0 @ERSpublications

Polypharmacy is frequent in COPD patients with acute exacerbation and is associated with severity and comorbidity http://ow.ly/wpwzG

Jesús Díez-Manglano ${ }^{1,2}$, José Barquero-Romero ${ }^{3}$, Pedro Almagro Mena ${ }^{4}$, Jesús Recio-Iglesias ${ }^{5}$, Javier Cabrera-Aguilar ${ }^{6}$, Francisco López-García ${ }^{7}$, Ramón Boixeda Viu ${ }^{8}$, Joan B. Soriano ${ }^{9}$ and the ECCO Study researchers ${ }^{10}$

${ }^{1}$ Internal Medicine Dept, Hospital Royo Villanova, Zaragoza, Spain. ${ }^{2}$ Aragon Health Sciences Institute, Zaragoza, Spain. ${ }^{3}$ Internal Medicine Dept, Hospital Tierra de Barros, Almendralejo, Badajoz, Spain. ${ }^{4}$ Internal Medicine Dept, Hospital Mutua de Tarrasa, Tarrasa, Barcelona, Spain. ${ }^{5}$ Internal Medicine Dept, Hospital Valle de Hebrón, Barcelona, Spain. ${ }^{6}$ Internal Medicine Dept, Hospital Gregorio Marañón, Madrid, Spain. ${ }^{7}$ Internal Medicine Dept, Hospital de la Vega Baja, Orihuela, Alicante, Spain. ${ }^{8}$ Internal Medicine Dept, Hospital de Mataró, Mataró, Barcelona, Spain. ${ }^{9}$ Fundación CaubetCimera, Bunyola, Mallorca, Spain. ${ }^{10}$ Working Group on COPD, Spanish Society of Internal Medicine.

Correspondence: Jesús Díez-Manglano, Hospital Royo Villanova, Avda. San Gregorio 30, 50015 Zaragoza, Spain. E-mail: jdiez@aragon.es

Received: Nov 072013 | Accepted after revision: April 222014 | First published online: May 152014

Conflict of interest: Disclosures can be found alongside the online version of this article at erj.ersjournals.com

\section{References}

1 Nielsen R, Johannessen A, Benediktsdottir B, et al. Present and future cost of COPD in Iceland and Norway: results of the BOLD study. Eur Respir J 2009; 34: 850-857. 
2 Jakobsen M, Anker N, Dollerup J, et al. Study on drug costs associated with COPD prescription medicine in Denmark. Clin Respir J 2013; 7: 328-337.

Blanchette CM, Dalal AA, Mapel D. Changes in COPD demographics and costs over 20 years. J Med Econ 2012; 15 : 1176-1182.

4 Corsonello A, Pedone C, Corica F, et al. Polypharmacy in elderly patients at discharge from the acute care hospital. Ther Clin Risk Manag 2007; 3: 197-203.

5 Hajjar ER, Hanlon JT, Artz MB, et al. Adverse drug reaction risk factors in older outpatients. Am J Geriatr Pharmacother 2003; 1: 82-89.

6 Gellad WF, Grenard JL, Marcum ZA. A systematic review of barriers to medication adherence in the elderly: looking beyond cost and regimen complexity. Am J Geriatr Pharmacother 2011; 9: 11-23.

7 Hovstadius B, Åstrand B, Persson U, et al. Acquisition cost of dispensed drugs in individuals with multiple medications - a register-based study in Sweden. Health Policy 2011; 101: 153-161.

8 Almagro P, López García F, Cabrera FJ, et al. Comorbidity and gender-related differences in patients hospitalized for COPD. The ECCO study. Respir Med 2010; 104: 253-259.

9 Vestbo J, Hurd SS, Agustí AG, et al. Global strategy for the diagnosis, management, and prevention of chronic obstructive pulmonary disease: GOLD executive summary. Am J Respir Crit Care Med 2013; 187: $347-365$.

10 Hajjar ER, Cafiero AC, Hanlon JT. Polypharmacy in elderly patients. Am J Geriatr Pharmacother 2007; 5: 345-351.

11 Nobili A, Marengoni A, Tettamanti M, et al. Association between clusters of diseases and polypharmacy in hospitalized elderly patients: results from the REPOSI study. Eur J Intern Med 2011; 22: 597-602.

12 Tinetti ME, Bogardus ST Jr, Agostini JV. Potential pitfalls of disease-specific guidelines for patients with multiple conditions. N Engl J Med 2004; 351: 2870-2874.

13 Travers J, Marsh S, Caldwell B, et al. External validity of randomized controlled trials in COPD. Respir Med 2007; 101: 1313-1320.

14 Holmes HM. Rational prescribing for patients with a reduced life expectancy. Clin Pharmacol Ther 2009; 85: $103-107$.

15 Cook DE, Sidel M, Belletti DA, et al. Review: clinical inertia in the management of chronic obstructive pulmonary disease. COPD 2012; 9: 73-80.

\section{Does a single Pseudomonas aeruginosa isolation predict COPD mortality?}

To the Editor:

Patients with chronic obstructive pulmonary disease (COPD) often suffer from acute exacerbations (AECOPD) of their disease, which have a significant impact on their health status [1]. Evidence suggests that $\sim 50 \%$ of these exacerbations are attributable to bacteria [2]. Pseudomonas aeruginosa can cause AECOPD and is associated with reduced survival in cystic fibrosis (CF) and bronchiectasis [3, 4]. Some studies have found that the presence of $P$. aeruginosa is also associated with mortality in COPD, but these findings have been based on patients hospitalised with exacerbations [5, 6] or those hospitalised with multidrug-resistant organisms [7].

The impact of $P$. aeruginosa identified in sputum from COPD outpatients is less clear but is an important issue for determining how aggressive strategies to attempt eradication should be. Therefore, we conducted a nested case-control study to investigate whether the isolation of $P$. aeruginosa in the sputum of a general COPD population was associated with long-term mortality.

We first identified all sputum specimen results from Royal Brompton Hospital (London, UK) microbiology records between 2000 and 2012. These were cross-correlated with patients listed on our COPD research audit database. The reason for obtaining a sputum culture was not recorded systematically, but included repeated exacerbations and deterioration in symptom severity and/or clinical status, as well as opportunistic collection from patients with chronic sputum production. The laboratory threshold for a culture to be considered P. aeruginosa positive was $200 \mathrm{CFU} \cdot \mathrm{mL}^{-1}$ (a semi-quantitative method). For patients with repeated positive sputum cultures the date of the first culture was recorded. Demographic variables, lung function measurements, gas transfer data, arterial blood gases and exacerbation frequency during the year prior to entering the study were recorded for all patients.

All analyses were performed using the Predictive Analytics Software (version 18; SPSS Inc., Chicago, IL, USA). Group comparisons were conducted utilising a t-test or the Chi-squared test as appropriate. Proportional Cox hazard analysis was utilised to assess: 1) the impact of parameters that differed between $P$. aeruginosa culture-positive and culture-negative groups and 2) the impact of antibiotic treatment on mortality. The proportionality hazard assumption was tested using partial residual plots (Schoenberg 